\title{
Optimization of Pulse Laser Annealing to Increase Sharpness of Implanted-junction Rectifier in Semi- conductor Heterostructure
}

\section{E. L. Pankratov*}

It has been recently shown that inhomogeneity of a semiconductor heterostructure leads to increasing of sharpness of diffusion-junction and implanted-junction rectifiers, which are formed in the semiconductor heterostructure. It has been also shown that together with increasing of the sharpness, homogeneity of impurity distribution in doped area increases. The both effect could be increased by formation of an inhomogeneous distribution of temperature (for example, by laser annealing). Some conditions on correlation between inhomogeneities of the semiconductor heterostructure and temperature distribution have been considered. Annealing time has been optimized for pulse laser annealing.

Keywords: Pulse laser; Implanted-juction rectifier; Heterostructure

Citation: E. L. Pankratov, "Optimization of Pulse Laser Annealing to Increase Sharpness of Implanted-junction Rectifier in Semiconductor Heterostructure”, Nano-Micro Lett. 2, 256-267 (2010). doi:10.3786/nml.v2i4.p256-267

Increasing performance and reliability of microelectronic devices and integrated circuits has attracted great interest recently. One way to increase performance of semiconductor devices is decreasing capacitance of $p$ - $n$-junctions [1,2]. The increase of homogeneity of dopant distribution in doped areas of a semiconductor structure allows to operate with higher current densities and to decrease local overheats or to decrease depth of $p$ - $n$-junction [1-3]. Another actual problem is the increase of exactness of theoretical description of dynamics of technological process. The increase leads to higher predictability of dopant dynamics and, as following, higher reproducibility of parameters of solid state electronic devices.

Different types of technological processes could be used for production $p-n$-junctions (see, for example, [1-5]). One of them is dopant diffusion into a semiconductor sample or in an epitaxial layer $(E L)$. Another one is ion implantation in the same cases. In this paper we consider a semiconductor heterostructure $(\mathrm{SH})$, which is presented in Fig. 1. The $S H$ consists of two layers. First of them is a substrate $(a \leq x \leq L)$ with diffusion coefficient $D_{2}$, thickness $L-a$ and known type of conductivity ( $n$ or $p$ ). The second layer of the $S H$ is an $E L(0 \leq x \leq a)$ with diffusion coefficient $D_{1}$ and thickness $a$. Let us consider a dopant, which is implanted across the boundary $x=0$ into the $E L$ to produce the opposite type of conductivity ( $p$ or $n$ ). At the time $t=0$ annealing of radiation defects is started with continuance $\Theta$. The annealing of radiation defects after production of the implanted-junction rectifier leads to a decrease of quantity of the defects and an increase of depth of the $p$ - $n$-junction. The increasing is unwanted, because the process leads to deviation of characteristics implanted-junction rectifier from scheduled values. It has been recently shown, that inhomogeneity of a $S H$ leads to increasing of sharpness of diffusion-junction (see, for example, [6,7]) and implanted-junction (see, for example, [8]) rectifiers, which are formed in the $S H$. It has been also shown, that together with increasing of the sharpness homogeneity of dopant distribution in doped area increases. To increase the both effects heating of surface region (the thickness of the heated surface region is approximately equal to the thickness $E L$ ) of the $S H$ attracting an 
interest. One way to produce the inhomogeneous distribution of temperature is pulse laser annealing. Another advantage of this type of annealing is local heating of the surface of the $S H$. The advantage is useful for production of elements of integrated circuits with decreasing spread of dopant across the interface of the $\mathrm{SH}$. Some theoretical analysis of spatiotemporal distribution of temperature during laser annealing has been done in previous works. However, the analysis has been done in simplified limiting cases.

The main aim of the present paper is to determine the conditions, which correspond to increasing of recently detected effect, i.e. to increase of the sharpness of the $p-n$-junction and the homogeneity of impurity concentration in doped areas at the same time. The accompanying aim is to develop mathematical approaches for analysis of dopant redistribution during annealing by laser pulses.

\section{Method of Solution}

Spatiotemporal distribution of dopant concentration in the considered $S H$ (see Fig. 1) has been described by the second Fick's law [1-3,5]

$$
\begin{aligned}
\frac{\partial C(x, t)}{\partial t} & =\frac{\partial}{\partial x}\left[D(x, T, V, C(x, t)) \frac{\partial C(x, t)}{\partial x}\right], \\
& =-\frac{\partial J_{C} C(x, t)}{\partial t}
\end{aligned}
$$

where $C(x, t)$ is the spatiotemporal distribution of dopant concentration. $J_{C}(x, t)$ is the spatiotemporal distribution of dopant flow. $D(x, T, V, C(x, t))$ is the diffusion coefficient of dopant in the $S H$. The diffusion coefficient depends on dynamical properties of dopant in materials of the $S H$, on temperature $T$ of annealing and on concentrations of radiation defects and dopant. It has been shown in Ref. [2] that in high-doped materials interaction between dopant atoms and point defects increases. If the point defects have nonzero charge $\gamma e$ with $e$ an elementary charge, then the interaction leads to concentrational dependence of the diffusion coefficient. The concentrational dependence of the diffusion coefficient could be approximated by the following function (see, for example, $[9,10]$ and [2])

$$
\begin{aligned}
& D(x, T, V, C(x, t))= \\
& D_{L}(x, T)\left[1+\varsigma \frac{V(x, t)}{V^{*}}\right]\left[1+\xi \frac{C^{\gamma}(x, t)}{P^{\gamma}(x, T)}\right]
\end{aligned}
$$

Here $V(x, t)$ and $V^{*}$ are spatiotemporal and equilibrium distributions of concentrations of vacancies. $P(x, T)$ is the limit of solubility of dopant in $S H$. The fitting parameters $\zeta, \xi$ and $\gamma$ depend on properties of layers of $S H$. Parameter $\zeta$ characterizes degree of radiation damage of SH. Parameter $\xi$ characterizes doping degree of $\mathrm{SH}$. Parameter $\gamma$ usually is equal to an integer value in the interval $\gamma \in[1,3]$ (see [2]). In the following let us consider the limiting case, when the number of different complexes (for example, complexes of defects) is negligible in comparison with the number of point defects. Spatiotemporal distribution of vacancies concentration is described by the following system of equation [3]

$$
\left\{\begin{aligned}
\frac{\partial I(x, t)}{\partial t}= & \frac{\partial}{\partial x}\left[D_{I}(x, T) \frac{\partial I(x, t)}{\partial x}\right]-k_{I, V}(x, T) \\
& {\left[I(x, t) V(x, t)-I^{*} V^{*}\right]=} \\
= & -\frac{\partial J_{I}(x, t)}{\partial t}-k_{I, V}(x, T) \\
\frac{\partial V(x, t)}{\partial t}= & \frac{\partial}{\partial x}\left[D_{V}(x, T) \frac{\partial V(x, t)}{\partial x}\right]-k_{I, V}(x, T) \\
& {\left[I(x, t) V(x, t)-I^{*} V^{*}\right] } \\
= & -\frac{\partial J_{V}(x, t)}{\partial t}-k_{I, V}(x, T) \\
& {\left[I(x, t) V(x, t)-I^{*} V^{*}\right] }
\end{aligned}\right.
$$

where $I(x, t)$ and $I^{*}$ are the spatiotemporal and the equilibrium distributions of interstitials, respectively. $J_{I}(x, t)$ and $J_{V}(x, t)$ are spatiotemporal distributions of flows of interstitials and vacancies, respectively. $D_{V}(x, T)$ and $D_{I}(x, T)$ are diffusion coefficients of vacancies and interstitials, respectively. $k_{I, V}(x, T)$ is the parameter of recombination of point defects. Spatiotemporal distribution of temperature could be estimated by using the second Fourier's law

$$
\begin{aligned}
c(T) \frac{\partial T(x, t)}{\partial t} & =\frac{\partial}{\partial x}\left[\lambda(x, T) \frac{\partial T(x, t)}{\partial x}\right]+p(x, t) \\
& =p(x, t)-\frac{\partial J_{T}(x, t)}{\partial x}
\end{aligned}
$$

where $c(T)$ is heat capacitance. For the most interesting (in our case) interval of values of temperature one can consider approximately constant value of heat capacitance $\left(c(T) \approx c_{a s s}\right)$. $\lambda(x, T)$ is the heat conduction coefficient. Temperature dependence of the heat conduction coefficient can be 
approximated by the following power law: $\lambda(x, T)=\lambda_{\text {ass }}(x)[1+\mu$ $\left.\left(T_{d} / T(x, t)\right)^{\varphi}\right]$ (see appropriate figures in [12]), $T_{d}$ is Debye temperature [12], $\mu$ and $\varphi$ are fitting parameters. $\alpha(x, T)=\lambda(x, T) / c(T)$ is thermal diffusivity. $p(x, t)$ is the bulk density of heat power, which is allocated in MS. The power could be approximated by the function: $p(x, t)=P_{0} \delta(x / L) \sin (\pi$ $t / \Theta), t \in[0, \Theta / 2], \delta(x / L)$ is Dirac $\delta$-function, $\Theta$ is the continuance of the laser pulse, $S$ is the lateral area of $S H$, and $P_{0}$ is the power of the laser pulse. $J_{T}(x, t)$ is spatiotemporal distribution of heat flow. The similar time dependence of power has been considered in [13]. However, the approximation considered in our work leads to simplification of analysis of mass and heat transport.

The Eqs. (1), (3) and (4) are complemented by the following boundary and initial conditions in the form

$J_{I}(0, t)=0, I(L, t)=0, I(x, 0)=f_{I}(x) ; J_{V}(0, t)=0, V(L, \mathrm{t})=0, V(x, 0)=f_{V}(x)$, $J_{C}(0, t)=0, C(L, t)=0$,

$C(x, 0)=f_{C}(x) ; J_{T}(0, t)=0, T(L, t)=T_{r}, T(x, 0)=f_{T}(x)$,

where $T_{r}$ is the equilibrium distribution of temperature, which coincides with room temperature.

First of all let us estimate spatiotemporal distribution of temperature. The parabolic equation has been transformed as where $\alpha_{\text {ass }}(x)=\lambda_{\text {ass }}(x) / c_{\text {ass }}(x)$.

$$
\begin{aligned}
& T(x, t)=T_{r}+\frac{1}{\mu T_{d}^{\varphi}} \int_{L}^{x} \frac{T^{\varphi}(v, t)}{\alpha_{\text {ass }}(v)} \int_{0}^{v} \frac{\partial T(u, t)}{\partial t} d u d v- \\
& \frac{1}{\mu T_{d}^{\varphi} c_{\text {ass }}} \int_{L}^{x} \frac{T^{\varphi}(v, t)}{\alpha_{\text {ass }}(v)} \int_{0}^{v} p(u, t) d u d v- \\
& \frac{T^{\varphi+1}(x, t)-T_{r}^{\varphi+1}}{\mu(\varphi+1) T_{d}^{\varphi}} \\
& I(x, t)=\int_{L}^{x} \frac{1}{D_{I}(v, T)} \int_{0}^{v} k_{I, V}(u, T) I(u, t) V(u, t) d u d v+ \\
& \int_{L}^{x} \frac{1}{D_{I}(v, T)} \int_{0}^{v} \frac{\partial I(u, t)}{\partial t} d u d v \\
& V(x, t)=\int_{L}^{x} \frac{1}{D_{V}(v, T)} \int_{0}^{v} k_{I, V}(u, T) I(u, t) V(u, t) d u d v+ \\
& \int_{L}^{x} \frac{1}{D_{V}(v, T)} \int_{0}^{v} \frac{\partial V(u, t)}{\partial t} d u d v \\
& C(x, t)=\int_{L}^{x} \frac{V^{*}}{D_{L}(v, T)\left[V^{*}+V(v, t)\right]} \int_{0}^{v} \frac{\partial C(u, t)}{\partial t} d u d v- \\
& \mu \int_{L}^{x} \frac{C^{\gamma}(v, t)}{P^{\gamma}(v, T)} \frac{\partial C(v, t)}{\partial v} d v
\end{aligned}
$$

Let us determine the solution of the system Eqs. (6) by averaging functional corrections (see, for example, [14].
Substitution of the average value of the functions $\rho(x, t)(\rho=\chi, T$; $\chi=I, V, L)$ and their partial derivatives in the right side of the Eqs. (6) instead of the considered functions gives us possibility to obtained the first-order approximations $\rho_{1}(x, t)$ of the functions $\rho$ $(x, t)$. To decrease steps of the iterative process, let us consider more accurate initial-order approximation (see, for example, [8]). As such approximation we consider the solutions of the equations of the system (6), which correspond to average values of diffusion coefficients $D_{0 L}, D_{0 I}$ and $D_{0 V}$, thermal diffusivity $\alpha_{0 a s s}$ and zero parameter of recombination. The solutions can be written in the form

$$
\begin{aligned}
& \tilde{I}(x, t)=\frac{2}{L} \sum_{n=0}^{\infty} F_{n I} c(x) e_{n I}(t), \\
& \tilde{V}(x, t)=\frac{2}{L} \sum_{n=0}^{\infty} F_{n V} c(x) e_{n V}(t), \\
& \tilde{C}(x, t)=\frac{2}{L} \sum_{n=0}^{\infty} F_{n C} c(x) e_{n C}(t), \\
& \tilde{T}(x, t)=T_{r}+\frac{2}{L} \sum_{n=1}^{\infty} F_{n T}(t) c_{n T}(x) e_{n T}(t),
\end{aligned}
$$

where

$$
\begin{aligned}
& F_{n \chi}=\int_{0}^{L} f_{\chi}(v) c_{n}(v) d v, \\
& F_{n T}(t)=\int_{0}^{t} e_{n T}(-\tau) \int_{0}^{L} c_{n}(v) \frac{p(v, \tau)}{C_{a s s}} d v d \tau, \\
& c_{n}(x)=\cos [\pi(n+0.5) x / L], e_{n T}(t)=\exp \left[-\pi^{2}(n+0.5)^{2} \alpha_{0 a s s} t / L^{2}\right], \\
& e_{n \chi}(t)=\exp \left[-\pi^{2}(n+0.5)^{2} D_{0 \chi} t / L^{2}\right] .
\end{aligned}
$$

Substitution of the Eqs. (7) into the right side of the equations of the system (6) instead of the functions $\rho(x, t)$ gives us possibility to obtain the first-order approximations (in the modified method of averaging of function corrections) of the appropriate functions. The algorithm is presented in details in [8] and will not be considered in this paper. The second-order approximations of the functions $\rho(x, t)$, by using the method of averaging of function corrections can be determined by using the standard procedure (see, for example, [8]), i.e. one shall substitute the sums $\alpha_{2 \rho}+\rho_{1}(x, t)$ instead of the functions $\rho(x, t)$ in the right side of the equations of the system (6). The substitution gives us possibility to obtained the second-order approximation of the functions $\rho_{2}(x, t)$. The algorithm is presented in details in $[8,14]$ and will not be considered in this paper.

The parameter $\alpha_{2 \rho}$ is determined by the following relation $[8,14]$, 


$$
\alpha_{2 \rho}=\frac{M_{i j \rho}-M_{i-1 j \rho}}{L \Theta},
$$

where

$$
M_{i j \rho}=\int_{0}^{\Theta} \int_{0}^{L} \rho_{i}^{j}(x, t) d x d t
$$

Furthemore let us analyze the dynamics of redistribution of dopant in the considered MS (see Fig. 1). The obtained analytical relations give us possibility to analyze the redistribution during annealing of dopant demonstratively. Using numerical approaches of the Eqs. (1), (3) and (4) leads to increase the exactness of the spatiotemporal distribution of dopant concentration.

\section{Discussion}

Let us analyze the dynamics of redistribution of dopant in the $S H$ (Fig. 1) for step-wise approximations of spatial distribution of diffusion coefficients of radiations defects and dopant and thermal diffusivity. In the case the approximations can be written as $\alpha_{a s s}(x)=\alpha_{a s s 1}[1(x)-1(x-a)]+\alpha_{a s s 2} 1(x-a)$ and $D_{\chi, L}(x)=D_{\chi, L 1}[1(x)-1(x-a)]+D_{\chi, L 2} 1(x-a)$, where $1(x)$ is the unit function; $D_{\chi, L 1}, D_{\chi, L 2}, \alpha_{a s s 1}$ and $\alpha_{a s s 2}$ are diffusion coefficient and thermal diffusivity of the $E L$ and substrate, respectively. Spatial distributions of dopant concentration for some values of annealing time and the difference between diffusion coefficients of $E L$ and the substrate are presented in Fig. 2. For simplification of analysis we consider the following normalization,

$$
\int_{0}^{L} C(x, t) d x=1 .
$$

Figure 2 shows that semi-insulating property of interface of $S H$ gives us possibility to increase sharpness of $p$ - $n$-junction and at the same time to increase homogeneity of dopant distribution

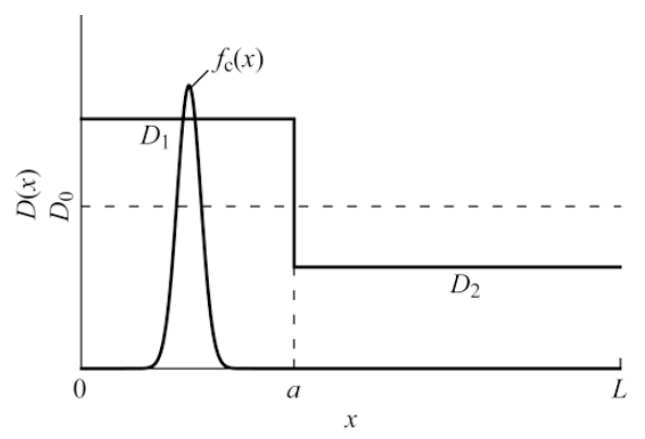

FIG. 1. Semiconductor heterostructure, which consist of an epitaxial layer $(x \in[0, a])$ and a substrate $(x \in[a, L])$.

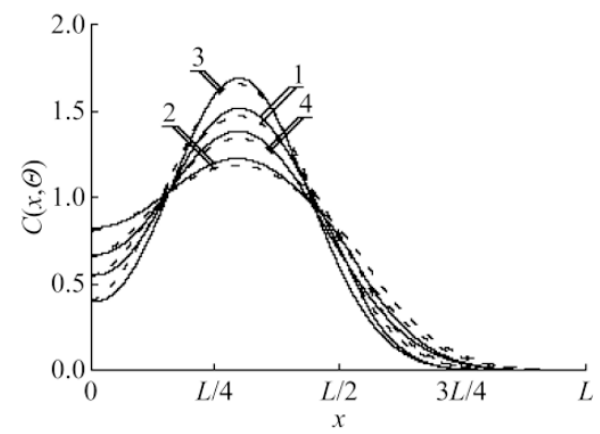

FIG. 2. Calculated distribution of dopant after annealing with continuance $\Theta=$ $0.0025 D_{0 L} / L^{2}$ (curves 1 and 3 ) and $\Theta=0.005 D_{0 L} / L^{2}$ (curves 2 and 4). Curves 1 and 2 are corresponding to spatial of distribution of dopant in homogeneous material. Curves 3 and 4 are correspond to spatial distribution of dopant in $\mathrm{SH}$ for $D_{1 L} / D_{2 L}=4$. Solid lines are analytical results. Dashed lines are numerical results. Coordinate of interface is equal to $a=L / 2$.

in $E L$. The effects increase at the same time with the increase of differences between properties of layers of $S H$. The increase of sharpness gives us possibility to decrease transit time of charge carriers through the $p$ - $n$-junction. The increase of homogeneity of dopant distribution leads to a decrease of local overheats in doped area. Dopant distributions on the figure are qualitatively similar with analogous distributions in Fig. 3 in reference [8]. But spreading of the distributions in the present paper is higher, because diffusion coefficient of dopant in $E L$ is larger in comparison with the same diffusion coefficient in [8] due to in homogeneity of distribution of temperature. Figure 2 in the present paper shows that inhomogeneity of the $S H$ leads to increasing the sharpness of $p$ - $n$-junction (if the junction was formed near the interface) and homogeneity of dopant distribution in doped area. The calculated spatial distribution of dopant and experimental one has been compared in Fig. 3. In this situation we obtain satisfactory agreement between calculated and measured results. The satisfactory agreement suggests our

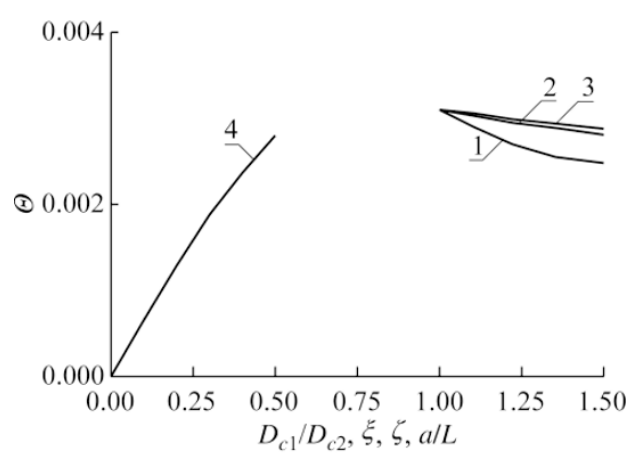

FIG. 3. Calculated distribution of dopant (solid line). Squares are experimental distribution of boron concentration in silicon (see [15]) for dose $F=2 \times 10^{15} \mathrm{~cm}^{-2}$. The boron distribution has been annealed by 20 laser pulses with continuance 23 $n s$, repetition rate 1 hertz and density of power $0.5 \mathrm{~J} / \mathrm{cm}^{2}$. 


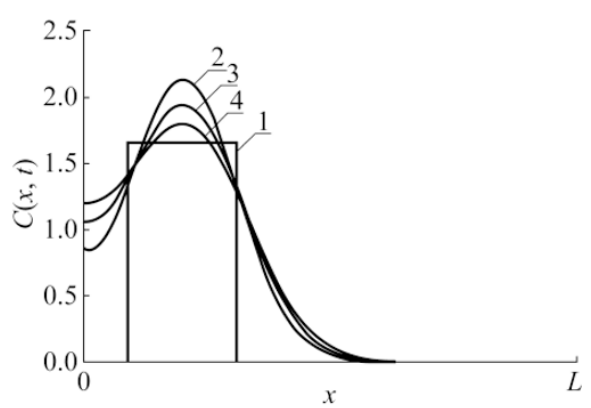

FIG. 4. Distributions of implanted dopant distributions (curves 1-3) for different values of annealing times. Increasing of number of curves corresponds to increasing of value of annealing time. Curve 4 is idealised approximation of curves $1-3$.

accurate choice of model for the present paper.

The increase of annealing time leads to the increase of the homogeneity of dopant distribution and to decrease of the sharpness of the $p$ - $n$-junction. Optimization of continuance of laser pulse leads to an increase of the effects at the same time. It should be noted, that two limiting cases of annealing of radiation defects could be considered. The first of them is the limiting case of large continuance of laser pulse (spreading of distribution of dopant is larger, than thickness of $E L$ ). The second of them is the limiting case of small continuance of laser pulse (spreading of distribution of dopant is smaller, than thickness of $E L$ ). Optimization of annealing time in the second limiting case is necessary, because the increasing of the continuance of laser pulse leads to shifting the $p$ - $n$-junction to the interface of the MS. Let us to use the earlier introduced criterion (see, for example, [6-8] for optimization of annealing time. To use the criterion to optimize the continuance of laser pulse we approximate spatiotemporal distribution of dopant concentration by step-wise function (see Fig. 4 and appropriate parts of Refs. [6-8]). To estimate the optimal continuance of laser pulse the mean squared error between the real spatiotemporal distribution of dopant concentration and step-wise approximation function should be minimized. Dependences of optimal continuance of laser pulse on several parameters are presented in Fig. 5. The figure shows that the increase of the thickness of the $E L$ leads to an increase of the compromise continuance of laser pulse. The increase of continuance of laser pulse is obtained due to the increase of continuance of dopant diffusion to interface between layers of $S H$. The increasing of the ratio $D_{1 L} / D_{2 L}$ and the parameter $\zeta$ leads to a decrease of the annealing time. The decrease could be obtained due to the increase of diffusion coefficient of $E L$, i.e. acceleration of dopant diffusion in the layer. At the same time dopant diffusion coefficient in the substrate decreases. The

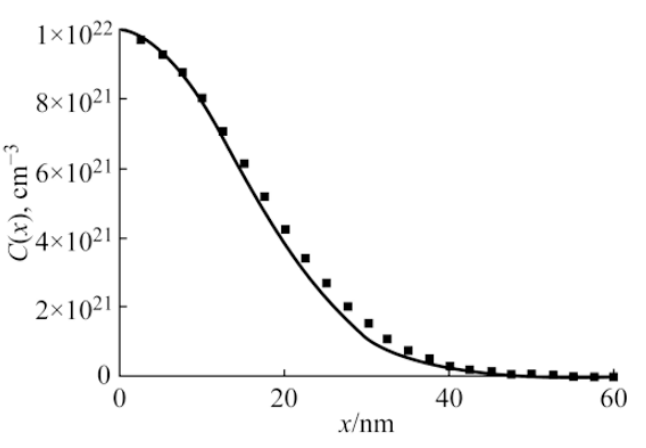

FIG. 5. Dependences compromise continuance of laser pulses $\Theta$ on some parameters of $S H$. Curve 1 is the dependence of $\Theta$ on the ratio $D_{1 L} / D_{2 L}$ for $\zeta$ $=0$ and $a=L / 2$. Curve 2 is the dependence of $\Theta$ on the parameter $\zeta$ for $D_{1 L} / D_{2 L}=1$ and $a=L / 2$. Curve 3 is the dependency of the $\Theta$ on the ration $a / L$ for $D_{1 L} / D_{2 L}=1$ and $\zeta=0$. Curve 4 is the dependence of $\Theta$ on the ratio $a / L$ for $D_{1 L} / D_{2 L}=1$ and $\zeta=0$.

deceleration of dopant diffusion in the substrate is the reason of the increase of sharpness of the $p$ - $n$-junction and the increase of homogeneity of dopant distribution in doped area. The obtained dependences of optimal annealing times are quantitatively differing from analogous dependences in [8] due to inhomogeneity of temperature distribution. It should be noted, that annealing by laser pulse with optimal continuance could be substituted by some laser pulses with smaller continuance, but with high frequency.

Dependences of optimal annealing time on several parameters are presented in Fig. 5. The figure shows that increasing of the thickness of the $E L$ leads to increasing of the compromise annealing time. Increasing of the ratio $D_{1 L} / D_{2 L}$ and the parameter $\zeta$ leads to decreasing of the annealing time. It should be noted, that annealing by laser pulse with optimal continuance could be substituted by some laser pulses with smaller continuance, but with high frequency. It should be noted, that optimal annealing time for the laser annealing case is smaller than optimal annealing time for the volumetric annealing case (see Fig. 5). It has been obtained that annealing times for Fig. 2 almost equal to optimal values of annealing times in Fig. 5. The difference could be explained by two reasons. The first of them is insufficient continuance of annealing in Fig. 2. The second one is finite exactness of mathematical approach.

\section{Conclusion}

In this paper we consider pulse laser annealing of radiation defects to increase sharpness of implanted-junction rectifier in semiconductor heterostructure. With the increase of the sharpness, homogeneity of dopant distribution in doped area increases. We optimized continuance of laser pulse to obtain 
maximal compromise between the increase of sharpness of the $p$ - $n$-junction and the increase of homogeneity of dopant distribution in doped area. The optimal continuance has been analyzed as a function of several parameters of heterostructure.

This work has been supported by grant of President of Russia (project № MK-548.2010.2).

\section{Received 11 October 2010; accepted 6 December 2010; published online 12 December 2010.}

\section{References}

1. A. B. Grebene, "Bipolar and MOS analogous integrated circuit design”, (John Wyley and Sons, New York, 1983).

2. Z. Y. Gotra, "Technology of microelectronic devices", Radio and communication, (Moscow, 1991, in Russian).

3. V. I. Lachin and N. S. Savelov, "Electronics", (Phoenix, Rostov-na-Donu, 2001, in Russian).

4. S. T. Sisianu, T. S. Sisianu and S. K. Railean, Semicond. 36, 581 (2002). doi:10.1134/1.1478552

5. T. Ahlgren, J. Likonen, J. Slotte, J. Raisanen, M. Rajatore and J. Keinonen, Phys. Rev. B. 56, 4597 (1997). doi:10.1103/PhysRevB.56.4597
6. E. L. Pankratov, Phys. Rev. B 72, 075201 (2005). doi:10. $\underline{\text { 1103/PhysRevB.72.075201 }}$

7. E. L. Pankratov and B. Spagnolo, Eur. Phys. J. B 46, 15 (2005). doi:10.1140/epjb/e2005-00233-1

8. E. L. Pankratov, Phys. Lett. A 372, 1897 (2008). doi:10.1016/j.physleta.2007.10.058

9. E. I. Zorin, P. V. Pavlov and D. I. Tetelbaum, "Ion doping of semiconductors", (Energiya, Moscow, 1975, in Russian).

10. H. Ryssel and I. Ruge, Ion implantation, (Teubner, Stuttgart, Germany, 1978).

11. P. M. Fahey, P. B. Griffin and J. D. Plummer, Rev. Mod. Phys. 61, 289 (1989). doi:10.1103/RevModPhys.61.289

12. K. V. Shalimova, "Physics of semiconductors", (Energoatomizdat, Moscow, 1985, in Russian).

13. V. I. Mazhukin, V. V. Nosov and U. Semmler, Mathematical modelling 12, 75 (2000).

14. Y. D. Sokolov, Applied Mechanics 1, 23 (1955) (in Russian).

15. K. K. Ong, K. L. Pey, P. S. Lee, A. T. S. Wee, X. C. Wang and Y. F. Chong, Appl. Phys. Lett. 89, 172111 (2006). doi:10.1063/1.2364834 


\section{Appendix}

Final analytical formulae describing curves at Fig. (2):

Relations for dopant concentration

$C_{2}(x, t)=\frac{2^{\gamma+1} \pi}{L^{\gamma+2}}\left(\frac{\pi^{2} D_{0 L}}{L^{2}}\right)^{\gamma-1} \sum_{n=0}^{\infty}(n+0.5) F_{n C} e_{n C}(t) \int_{L}^{x} \int_{L}^{v} \frac{V^{*}}{\left[V^{*}+V_{2}(w, t)\right]} \int_{0}^{w}\left[\sum_{n=0}^{\infty} n^{2} F_{n C} c_{n}(u) e_{n C}(t)\right]^{\gamma-1} \times$

$\times \mu \gamma \frac{d u d w}{D_{c}\left(v, T_{2}\right)} \frac{s_{n}(v) d v}{P^{\gamma}\left(v, T_{2}\right)}+D_{0 L} \frac{2 \pi}{L^{2}} \sum_{n=0}^{\infty}(n+0.5) F_{n C} \int_{L}^{x} \frac{V^{*}}{\left[V^{*}+V_{2}(v, t)\right]} \int_{0}^{v} \int_{L}^{w} \frac{V^{*} S_{n+0.5}(u)}{D_{c}\left(u, T_{2}\right)\left[V^{*}+V_{2}(u, t)\right]^{2}} \times$

$\times \frac{\partial V_{2}(u, t)}{\partial u} \frac{d u d w d v}{D_{c}\left(v, T_{2}\right)}-\mu \int_{L}^{x}\left\{\mu \frac{2^{\gamma+1} \pi}{L^{\gamma+2}} \sum_{n=0}^{\infty}(n+0.5) F_{n C} e_{n C}(t) \int_{L}^{v} \frac{s_{n}(u)}{P^{\gamma}\left(v, T_{2}\right)}\left[\sum_{n=0}^{\infty} F_{n C} c_{n}(u) e_{n C}(t)\right]^{\gamma} d u-\right.$

$\left.-D_{0 L} \frac{2 \pi}{L^{2}} \sum_{n=0}^{\infty}(n+0.5) F_{n C} \int_{L}^{v} \frac{V^{*} S_{n+0.5}(u) d u}{D_{C}\left(u, T_{2}\right)\left[V^{*}+V_{2}(u, t)\right]}+\alpha_{2 C}\right\}^{\gamma}\left\{-\mu \gamma D_{0 L} \frac{2^{\gamma+1} \pi^{3}}{L^{\gamma+4}} \sum_{n=0}^{\infty}(n+0.5) e_{n C}(t) \times\right.$

$\times F_{n C} \int_{L}^{v} \frac{s_{n}(w)}{P^{\gamma}\left(w, T_{2}\right)}\left[\sum_{n=0}^{\infty} F_{n C} c_{n}(w) e_{n C}(t)\right]^{\gamma-1} d w+D_{0 L} \frac{2 \pi}{L^{2}} \sum_{n=0}^{\infty}(n+0.5) F_{n C} \int_{L}^{v} \frac{\partial V_{2}(w, t)}{\partial u} \frac{V^{*} S_{n+0.5}(w)}{\left[V^{*}+V_{2}(w, t)\right]^{2}} \times$

$\times \frac{d w}{D_{C}^{2}\left(w, T_{2}\right)} \frac{d v}{P^{\gamma}\left(v, T_{2}\right)}$

where $\alpha_{2 C}$ is determined by solving the following equation for concrete values of parameter $\gamma$

$$
\begin{aligned}
& \alpha_{2 C}=\frac{\mu}{L \Theta} \int_{0}^{\Theta} \int_{0}^{L}\left\{\alpha_{2 C}+\mu \frac{2^{\gamma+1} \pi}{L^{\gamma+2}} \sum_{n=0}^{\infty}(n+0.5) F_{n C} e_{n C}(t) \int_{L}^{x} \frac{s_{n}(v)}{P^{\gamma}\left(v, T_{2}\right)}\left[\sum_{m=0}^{\infty} F_{m C} c_{m}(v) e_{m C}(t)\right]^{\gamma} d v-\right. \\
& \left.-D_{0 C} \frac{2 \pi}{L^{2}} \sum_{n=0}^{\infty}(n+0.5) F_{n C} \int_{L}^{x} \frac{V^{*} S_{n+0.5}(v) d v}{D_{c}\left(v, T_{2}\right)\left[V^{*}+V_{2}(v, t)\right]}\right\}^{\gamma}\left\{D_{0 L} \frac{2 \pi}{L^{2}} \sum_{n=0}^{\infty}(n+0.5) F_{n C} \int_{L}^{x} \frac{S_{n+0.5}(v)}{D_{c}\left(v, T_{2}\right)} \times\right. \\
& \times \frac{\partial V_{2}(v, t)}{\partial t} \frac{V^{*} d v}{\left[V^{*}+V_{2}(v, t)\right]^{2}}-\mu D_{0 L} \frac{2^{\gamma+1} \pi^{3}}{L^{\gamma+4}} \sum_{n=0}^{\infty} n^{2}(n+0.5) e_{n C}(t) \int_{L}^{x}\left[\sum_{m=0}^{\infty} F_{m C} c_{m}(x) e_{m C}(t)\right]^{\gamma} \times
\end{aligned}
$$

$\times \frac{s_{n}(v) d v}{P^{\gamma}\left(v, T_{2}\right)} \frac{x d x d t}{P^{\gamma}\left(x, T_{2}\right)} F_{n C}+\mu D_{0 L} \frac{2^{\gamma+1} \pi^{3}}{\Theta L^{\gamma+5}} \int_{0}^{\Theta} \int_{0}^{L} \frac{x V^{*}}{D_{c}\left(x, T_{2}\right)\left[V^{*}+V_{2}(x, t)\right]} \int_{0}^{x} \sum_{n=0}^{\infty}(n+0.5) e_{n C}(t) \times$

$\times F_{n C} \int_{L}^{w} \frac{s_{n}(v)}{P^{\gamma}\left(v, T_{2}\right)}\left[\sum_{n=0}^{\infty} F_{n C} c_{n}(x) e_{n C}(t)\right]^{\gamma} d v d w d x d t+2 \pi \frac{D_{0 L}}{L^{3} \Theta}\left(V^{*}\right)^{2} \sum_{n=0}^{\infty}(n+0.5) F_{n C} \times$

$\times \int_{0}^{\Theta} \int_{0}^{L} \frac{x}{D_{c}\left(x, T_{2}\right)\left[V^{*}+V_{2}(x, t)\right]} \int_{0}^{x} \int_{L}^{v} \frac{s_{n+0.5}(u) d u d v d x d t}{D_{c}\left(u, T_{2}\right)\left[V^{*}+V_{2}(u, t)\right]}-\mu \frac{2^{\gamma+1} \pi}{\Theta L^{\gamma+3}} \sum_{n=0}^{\infty}(n+0.5) F_{n C}\left\{\int_{0}^{\Theta} e_{n C}(t) \times\right.$ 
$\left.\times \int_{0}^{L}(L-x)\left[\sum_{n=0}^{\infty} F_{n C} c_{n}(x) e_{n C}(t)\right]^{\gamma} \frac{s_{n}(x) d x}{P^{\gamma}\left(x, T_{2}\right)} d t-L \int_{0}^{\Theta} e_{n C}(t) \int_{0}^{L}\left[\sum_{n=0}^{\infty} F_{n C} c_{n}(x) e_{n C}(t)\right]^{\gamma} \frac{s_{n}(v) d v}{P^{\gamma}\left(v, T_{2}\right)} d t\right\} ;$

Relations for temperature

$T_{2}(x, t)=\frac{1}{\mu T_{d}^{\varphi}} \int_{L}^{x}\left\{\alpha_{2 T}+T_{r}-\pi \frac{2 \alpha_{a s s} 0}{\mu T_{d}^{\varphi} L} \sum_{n=0}^{\infty}(n+0.5) \int_{0}^{\Theta} e_{n T}(-\vartheta) \int_{0}^{L} c_{n}(u) \frac{p(u, \vartheta)}{c_{a s s}} d u d \vartheta \int_{L}^{x} \frac{s_{n}(v)}{\alpha_{a s s}(v)}\left[T_{r}+\right.\right.$

$\left.+\frac{2}{L} \sum_{n=0}^{\infty} c_{n}(v) e_{n T}(t) \int_{0}^{\Theta} e_{n T}(-\vartheta) \int_{0}^{L} c_{n}(u) \frac{p(u, \vartheta)}{c_{a s s}} d u d \vartheta\right]^{\varphi} d v-\frac{1}{\mu T_{d}^{\varphi}} \int_{L}^{x} \frac{1}{\alpha_{a s s}(v)}\left[T_{r}+\frac{2}{L} \sum_{n=0}^{\infty} c_{n}(v) e_{n T}(t) \times\right.$

$\left.\times \int_{0}^{\Theta} e_{n T}(-\vartheta) \int_{0}^{L} c_{n}(u) \frac{p(u, \vartheta)}{c_{a s s}} d u d \vartheta\right]_{0}^{\varphi} \int_{0}^{v} \frac{p(u, t)}{c_{a s s}} d u d v-\frac{1}{\mu(\varphi+1) T_{d}^{\varphi}}\left[\frac{2}{L} \sum_{n=0}^{\infty} c_{n}(x) e_{n T}(t) \int_{0}^{\Theta} e_{n T}(-\vartheta) \times\right.$

$\left.\left.\times \int_{0}^{L} c_{n}(u) \frac{p(u, \vartheta)}{c_{a s s}} d u d \vartheta+T_{r}\right]^{\varphi+1}+\frac{T_{r}^{\varphi+1}}{\mu(\varphi+1) T_{d}^{\varphi}}\right\}^{\varphi} \int_{0}^{v}\left\{\pi^{2} \frac{8 \varphi \alpha_{a s s}^{2}}{\mu T_{d}^{\varphi} L^{4}} \sum_{n=0}^{\infty}(n+0.5) \int_{0}^{\ominus} e_{n T}(-\vartheta) \int_{0}^{L} \frac{p(u, \vartheta)}{c_{a s s}} \times\right.$

$\times c_{n}(u) d u d \vartheta \int_{L}^{w} \frac{S_{n}(v)}{\alpha_{a s s}(v)}\left[T_{r}+\frac{2}{L} \sum_{n=0}^{\infty} c_{n}(v) e_{n T}(t) \int_{0}^{\ominus} e_{n T}(-\vartheta) \int_{0}^{L} c_{n}(u) \frac{p(u, \vartheta)}{c_{a s s}} d u d \vartheta\right]^{\rho-1} \sum_{m=0}^{\infty} c_{m}(v) e_{m T}(t) \times$

$\times m^{2} \int_{0}^{\ominus} e_{m T}(-\vartheta) \int_{0}^{L} c_{m}(u) \frac{p(u, \vartheta)}{c_{a s s}} d u d \vartheta d v-\frac{1}{\mu T_{d}^{\varphi}} \int_{L}^{w} \frac{1}{\alpha_{a s s}(v)}\left[\frac{2}{L} \sum_{n=0}^{\infty} c_{n}(v) e_{n T}(t) \int_{0}^{\ominus} e_{n T}(-\vartheta) \int_{0}^{L} \frac{p(u, \vartheta)}{c_{a s s}} \times\right.$

$\left.\times c_{n}(u) d u d \vartheta+T_{r}\right]^{\varphi} \int_{0}^{v} \frac{p(u, t)}{c_{a s s}} d u d v+\frac{2}{\mu}\left[T_{r}+\frac{2}{L} \sum_{n=0}^{\infty} c_{n}(w) e_{n T}(t) \int_{0}^{\ominus} e_{n T}(-\vartheta) \int_{0}^{L} c_{n}(u) \frac{p(u, \vartheta)}{c_{a s s}} d u d \vartheta\right]^{\varphi} \times$

$\left.\times \frac{\pi^{2}}{L^{3} T_{d}^{\varphi}} \sum_{m=0}^{\infty} m^{2} c_{m}(w) e_{m T}(t) \int_{0}^{\ominus} e_{m T}(-\vartheta) \int_{0}^{L} c_{m}(u) \frac{p(u, \vartheta)}{c_{a s s}} d u d \vartheta\right\} \frac{d w d v}{\alpha_{a s s}(v)}-\frac{1}{\mu(\varphi+1) T_{d}^{\varphi}}\left\{\alpha_{2 T}-\frac{2 \alpha_{a s s}}{\mu T_{d}^{\varphi} L} \times\right.$

$\times \pi \sum_{n=0}^{\infty}(n+0.5) \int_{0}^{\Theta} e_{n T}(-\vartheta) \int_{0}^{L} c_{n}(u) \frac{p(u, \vartheta)}{c_{a s s}} d u d \vartheta \int_{L}^{x} \frac{s_{n}(v)}{\alpha_{a s s}(v)}\left[T_{r}+\frac{2}{L} \sum_{n=0}^{\infty} c_{n}(v) e_{n T}(t) \int_{0}^{\Theta} \int_{0}^{L} c_{n}(u) \frac{p(u, \vartheta)}{c_{a s s}} d u \times\right.$

$\left.\times e_{n T}(-\vartheta) d \vartheta\right]^{\varphi} d v+T_{r}-\frac{1}{\mu T_{d}^{\varphi}} \int_{L}^{x} \frac{1}{\alpha_{a s s}(v)}\left[T_{r}+\frac{2}{L} \sum_{n=0}^{\infty} c_{n}(v) e_{n T}(t) \int_{0}^{\ominus} e_{n T}(-\vartheta) \int_{0}^{L} c_{n}(u) \frac{p(u, \vartheta)}{c_{a s s}} d u d \vartheta\right] \times$

$\times \int_{0}^{v} \frac{p(u, t)}{c_{a s s}} d u d v-\frac{1}{\mu(\varphi+1) T_{d}^{\varphi}}\left[T_{r}+\frac{2}{L} \sum_{n=0}^{\infty} c_{n}(x) e_{n T}(t) \int_{0}^{\ominus} e_{n T}(-\vartheta) \int_{0}^{L} c_{n}(u) \frac{p(u, \vartheta)}{c_{a s s}} d u d \vartheta\right]^{\varphi+1}+\frac{T_{r}^{\varphi+1}}{(\varphi+1)} \times$

$\left.\times \frac{1}{\mu T_{d}^{\varphi}}\right\}^{\varphi+1}+T_{r}\left[1+\frac{T_{r}^{\varphi}}{\mu(\varphi+1) T_{d}^{\varphi}}\right]-\int_{L}^{x}\left\{\alpha_{2 T}-\pi \frac{2 \alpha_{a s s}}{\mu T_{d}^{\varphi} L} \sum_{n=0}^{\infty}(n+0.5) \int_{0}^{\Theta} e_{n T}(-\vartheta) \int_{0}^{L} c_{n}(u) \frac{p(u, \vartheta)}{c_{a s s}} d u d \vartheta \times\right.$ 
Nano-Micro Lett. 2, 256-267 (2010)

264

E. L. Pankratov

$$
\begin{aligned}
& \times \int_{L}^{x} \frac{S_{n}(v)}{\alpha_{a s s}(v)}\left[T_{r}+\frac{2}{L} \sum_{n=0}^{\infty} c_{n}(v) e_{n T}(t) \int_{0}^{\ominus} e_{n T}(-\vartheta) \int_{0}^{L} c_{n}(u) \frac{p(u, \vartheta)}{c_{a s s}} d u d \vartheta\right]^{\varphi} d v+T_{r}-\frac{1}{\mu T_{d}^{\varphi}} \int_{L}^{w} \frac{1}{\alpha_{a s s}(v)}\left[T_{r}+\right. \\
& \left.+\frac{2}{L} \sum_{n=0}^{\infty} c_{n}(v) e_{n T}(t) \int_{0}^{\ominus} e_{n T}(-\vartheta) \int_{0}^{L} c_{n}(u) \frac{p(u, \vartheta)}{c_{a s s}} d u d \vartheta\right]_{0}^{\varphi} \int_{0}^{v} \frac{p(u, t)}{c_{a s s}} d u d v-\frac{1}{\mu(\varphi+1) T_{d}^{\varphi}}\left[T_{r}+\frac{2}{L} \sum_{n=0}^{\infty} c_{n}(x) \times\right. \\
& \left.\left.\times e_{n T}(t) \int_{0}^{\ominus} e_{n T}(-\vartheta) \int_{0}^{L} \frac{p(u, \vartheta)}{c_{a s s}} c_{n}(u) d u d \vartheta\right]^{\varphi+1}+\frac{T_{r}^{\varphi+1}}{\mu(\varphi+1) T_{d}^{\varphi}}\right\}_{0}^{\varphi} \frac{p(u, t)}{c_{a s s}} d u \frac{d w}{\alpha_{a s s}(w)} \frac{1}{\mu T_{d}^{\varphi}},
\end{aligned}
$$

where $\alpha_{2 T}$ is determined by solving the following equation for concrete values of parameter $\varphi$

$$
\begin{aligned}
& \alpha_{2 T}=T_{r}\left[1+\frac{T_{r}^{\varphi}}{\mu(\varphi+1) T_{d}^{\varphi}}\right]+\frac{1}{\mu T_{d}^{\varphi} L \Theta} \int_{0}^{\Theta} \int_{0}^{L}\left\{\alpha_{2 T}-\pi \frac{2 \alpha_{a s s}}{\mu T_{d}^{\varphi} L} \sum_{n=0}^{\infty}(n+0.5) \int_{0}^{\Theta} e_{n T}(-\vartheta) \int_{0}^{L} c_{n}(u) \frac{p(u, \vartheta)}{c_{a s s}} d u d \vartheta \times\right. \\
& \times \int_{L}^{x} \frac{s_{n}(v)}{\alpha_{a s s}(v)}\left[T_{r}+\frac{2}{L} \sum_{n=0}^{\infty} c_{n}(v) e_{n T}(t) \int_{0}^{\ominus} e_{n T}(-\vartheta) \int_{0}^{L} c_{n}(u) \frac{p(u, \vartheta)}{c_{a s s}} d u d \vartheta\right]^{\varphi} d v-T_{r}-\frac{1}{\mu T_{d}^{\varphi}} \int_{L}^{x} \frac{1}{\alpha_{a s s}(v)}\left[\frac{2}{L} \sum_{n=0}^{\infty} c_{n}(v) \times\right. \\
& \left.\times e_{n T}(t) \int_{0}^{\Theta} e_{n T}(-\vartheta) \int_{0}^{L} c_{n}(u) \frac{p(u, \vartheta)}{c_{a s s}} d u d \vartheta+T_{r}\right]^{\varphi} d v-\frac{1}{\mu T_{d}^{\varphi}} \int_{L}^{x} \frac{1}{\alpha_{a s s}(v)}\left[\frac{2}{L} \sum_{n=0}^{\infty} c_{n}(v) e_{n T}(t) \int_{0}^{\Theta} e_{n T}(-\vartheta) \int_{0}^{L} c_{n}(u) \times\right. \\
& \left.\times \int_{0}^{L} c_{n}(u) \frac{p(u, \vartheta)}{c_{a s s}} d u d \vartheta\right]_{0}^{\varphi} \int_{0}^{v} \frac{p(u, t)}{c_{a s s}} d u d v-\frac{1}{\mu(\varphi+1) T_{d}^{\varphi}}\left[T_{r}+\frac{2}{L} \sum_{n=0}^{\infty} c_{n}(x) e_{n T}(t) \int_{0}^{\ominus} e_{n T}(-\vartheta) \int_{0}^{L} \frac{p(u, \vartheta)}{c_{a s s}} \times\right. \\
& \left.\left.\times c_{n}(u) d u d \vartheta\right]^{\varphi+1}+\frac{T_{r}^{\varphi+1}}{\mu(\varphi+1) T_{d}^{\varphi}}\right\}^{\varphi} \int_{0}^{x}\left\{4 \pi^{3} \frac{\varphi \alpha_{a s s}^{2}}{\mu T_{d}^{\varphi} L^{4}} \sum_{n=0}^{\infty} \int_{0}^{\ominus} e_{n T}(-\vartheta) \int_{0}^{L} c_{n}(u) \frac{p(u, \vartheta)}{c_{a s s}} d u d \vartheta \int_{L}^{x} \frac{s_{n}(v)}{\alpha_{a s s}(v)} \times\right. \\
& \times(n+0.5)\left[T_{r}+\frac{2}{L} \sum_{n=0}^{\infty} c_{n}(v) e_{n T}(t) \int_{0}^{\Theta} e_{n T}(-\vartheta) \int_{0}^{L} c_{n}(u) \frac{p(u, \vartheta)}{c_{a s s}} d u d \vartheta\right]^{\varphi-1} \sum_{m=0}^{\infty} m^{2} c_{m}(v) e_{m T}(t) \int_{0}^{\ominus} e_{m T}(-\vartheta) \times \\
& \times \int_{0}^{L} c_{m}(u) \frac{p(u, \vartheta)}{c_{a s s}} d u d \vartheta d v-\frac{1}{\mu T_{d}^{\varphi}} \int_{L}^{x} \frac{1}{\alpha_{a s s}(v)}\left[T_{r}+\sum_{n=0}^{\infty} c_{n}(v) e_{n T}(t) \int_{0}^{\Theta} e_{n T}(-\vartheta) \int_{0}^{L} c_{n}(u) \frac{p(u, \vartheta)}{c_{a s s}} d u d \vartheta \times\right. \\
& \left.\times \frac{2}{L}\right]^{\varphi-1} \sum_{m=0}^{\infty} m^{2} c_{m}(v) e_{m T}(t) \int_{0}^{\Theta} e_{m T}(-\vartheta) \int_{0}^{L} c_{m}(u) \frac{p(u, \vartheta)}{c_{a s s}} d u d \vartheta-\frac{1}{\mu T_{d}^{\varphi}}\left[T_{r}+\sum_{n=0}^{\infty} c_{n}(x) e_{n T}(t) \int_{0}^{\Theta} e_{n T}(-\vartheta) \times\right. \\
& \left.\left.\times \frac{2}{L} \int_{0}^{L} \frac{p(u, \vartheta)}{c_{a s s}} c_{n}(u) d u d \vartheta\right]_{m=0}^{\varphi} \sum^{2} c_{m}(v) e_{m T}(t) \int_{0}^{\Theta} e_{m T}(-\vartheta) \int_{0}^{L} c_{m}(u) \frac{p(u, \vartheta)}{c_{a s s}} d u d \vartheta\right\} d x d t+\frac{1}{\mu L \Theta T_{d}^{\varphi}} \times \\
& \times \int_{0}^{\Theta} \int_{0}^{L}\left\{\alpha_{2 T}+T_{r}-\pi \frac{2 \alpha_{a s s} 0}{\mu T_{d}^{\varphi} L} \sum_{n=0}^{\infty}(n+0.5) \int_{0}^{\Theta} e_{n T}(-\vartheta) \int_{0}^{L} c_{n}(u) \frac{p(u, \vartheta)}{c_{a s s}} d u d \vartheta \int_{L}^{x} \frac{s_{n}(v)}{\alpha_{a s s}(v)}\left[T_{r}+\frac{2}{L} \sum_{n=0}^{\infty} c_{n}(v) e_{n T}(t) \times\right.\right.
\end{aligned}
$$

DOI:10.3786/nml.v2i4.p256-267

http://www.nmletters.org 


$$
\begin{aligned}
& \left.\times \int_{0}^{\Theta} e_{n T}(-\vartheta) \int_{0}^{L} c_{n}(u) \frac{p(u, \vartheta)}{c_{a s s}} d u d \vartheta\right]^{\varphi} d v-\frac{1}{\mu T_{d}^{\varphi}} \int_{L}^{x} \frac{1}{\alpha_{a s s}(v)}\left[T_{r}+\frac{2}{L} \sum_{n=0}^{\infty} \int_{0}^{\Theta} e_{n T}(-\vartheta) \int_{0}^{L} c_{n}(u) \frac{p(u, \vartheta)}{c_{a s s}} d u d \vartheta \times\right. \\
& \left.\times c_{n}(v) e_{n T}(t)\right]^{\varphi} \int_{0}^{v} \frac{p(u, t)}{c_{a s s}} d u d v-\frac{1}{\mu(\varphi+1)}\left[T_{r}+\frac{2}{L} \sum_{n=0}^{\infty} c_{n}(x) e_{n T}(t) \int_{0}^{\ominus} e_{n T}(-\vartheta) \int_{0}^{L} c_{n}(u) \frac{p(u, \vartheta)}{c_{a s s}} d u d \vartheta\right]^{\varphi+1} \times \\
& \left.\times \frac{1}{T_{d}^{\varphi}}+\frac{T_{r}^{\varphi+1}}{\mu(\varphi+1) T_{d}^{\varphi}}\right\}^{\varphi} \int_{0}^{x} \frac{p(v, t)}{c_{a s s}} d v \frac{x d x d t}{\alpha_{a s s}(x)}-\frac{1}{L \Theta} \int_{0}^{\Theta} \int_{0}^{L}\left\{\alpha_{2 T}+T_{r}-\pi \frac{2 \alpha_{a s 0}}{\mu T_{d}^{\varphi} L} \sum_{n=0}^{\infty}(n+0.5) \int_{0}^{\Theta} e_{n T}(-\vartheta) \int_{0}^{L} c_{n}(u) \times\right. \\
& \times \frac{p(u, \vartheta)}{c_{a s s}} d u d \vartheta \int_{L}^{x} \frac{s_{n}(v)}{\alpha_{a s s}(v)}\left[T_{r}+\frac{2}{L} \sum_{n=0}^{\infty} c_{n}(v) e_{n T}(t) \int_{0}^{\Theta} e_{n T}(-\vartheta) \int_{0}^{L} c_{n}(u) \frac{p(u, \vartheta)}{c_{a s s}} d u d \vartheta\right]^{\varphi} d v-\frac{1}{\mu T_{d}^{\varphi}} \int_{L}^{x} \frac{1}{\alpha_{a s s}(v)} \times \\
& \times\left[T_{r}+\frac{2}{L} \sum_{n=0}^{\infty} c_{n}(v) e_{n T}(t) \int_{0}^{\ominus} e_{n T}(-\vartheta) \int_{0}^{L} c_{n}(u) \frac{p(u, \vartheta)}{c_{a s s}} d u d \vartheta\right]_{0}^{\varphi} \int_{0}^{v} \frac{p(u, t)}{c_{a s s}} d u d v-\frac{1}{(\varphi+1) T_{d}^{\varphi}}\left[T_{r}+\frac{2}{L} \sum_{n=0}^{\infty} c_{n}(x) \times\right. \\
& \left.\left.\times e_{n T}(t) \int_{0}^{\Theta} e_{n T}(-\vartheta) \int_{0}^{L} c_{n}(u) \frac{p(u, \vartheta)}{c_{a s s}} d u d \vartheta\right]^{\varphi+1} \frac{1}{\mu}+\frac{T_{r}^{\varphi+1}}{\mu(\varphi+1) T_{d}^{\varphi}}\right\}^{\varphi+1} d x d t-\frac{1}{\mu L \Theta(\varphi+1) T_{d}^{\varphi}} \int_{0}^{\Theta} \int_{0}^{L}\left\{\alpha_{2 T}+T_{r}-\right. \\
& -\pi \frac{2 \alpha_{a s s} 0}{\mu T_{d}^{\varphi} L} \sum_{n=0}^{\infty}(n+0.5) \int_{0}^{\ominus} e_{n T}(-\vartheta) \int_{0}^{L} c_{n}(u) \frac{p(u, \vartheta)}{c_{a s s}} d u d \vartheta \int_{L}^{x} \frac{s_{n}(v)}{\alpha_{a s s}(v)}\left[T_{r}+\frac{2}{L} \sum_{n=0}^{\infty} c_{n}(v) e_{n T}(t) \int_{0}^{\ominus} e_{n T}(-\vartheta) \int_{0}^{L} c_{n}(u) \times\right. \\
& \left.\times \frac{p(u, \vartheta)}{c_{a s s}} d u d \vartheta\right]^{\varphi} d v-\frac{1}{\mu} \int_{L}^{x}\left[T_{r}+\frac{2}{L} \sum_{n=0}^{\infty} c_{n}(v) e_{n T}(t) \int_{0}^{\ominus} e_{n T}(-\vartheta) \int_{0}^{L} c_{n}(u) \frac{p(u, \vartheta)}{c_{a s s}} d u d \vartheta\right]_{0}^{\varphi} \int_{0}^{v} \frac{p(u, t)}{c_{a s s}} d u \frac{d v}{\alpha_{a s s}(v)} \times \\
& \left.\times \frac{1}{T_{d}^{\varphi}}-\frac{1}{\mu(\varphi+1) T_{d}^{\varphi}}\left[T_{r}+\frac{2}{L} \sum_{n=0}^{\infty} c_{n}(x) e_{n T}(t) \int_{0}^{\Theta} e_{n T}(-\vartheta) \int_{0}^{L} c_{n}(u) \frac{p(u, \vartheta)}{c_{a s s}} d u d \vartheta\right]^{\varphi+1}+\frac{T_{r}^{\varphi+1}}{\mu(\varphi+1) T_{d}^{\varphi}}\right\}^{\varphi+1} d x d t
\end{aligned}
$$

Relations for temperature

$$
\begin{aligned}
& I_{2}(x, t)=-D_{0 I} \frac{4 \pi^{2}}{L^{4}} \int_{L}^{x} \int_{0}^{v} \int_{0}^{w} k_{I, V}(y, T) \sum_{n=0}^{\infty} n^{2} F_{n I} c_{n}(y) e_{n I}(t) \sum_{m=0}^{\infty} F_{m V} c_{m}(y) e_{m V}(t) d y \frac{w d w}{D_{I}\left(w, T_{2}\right)} \frac{d u d v}{D_{I}\left(v, T_{2}\right)}- \\
& -D_{0 V} \frac{4 \pi^{2}}{L^{4}} \int_{L}^{x} \int_{0}^{v} \int_{0}^{w} k_{I, V}\left(y, T_{2}\right) \sum_{n=0}^{\infty} F_{n I} c_{n}(y) e_{n I}(t) \sum_{m=0}^{\infty} m^{2} F_{m V} c_{m}(y) e_{m V}(t) d y \frac{w d w}{D_{I}\left(w, T_{2}\right)} \frac{d u d v}{D_{I}\left(v, T_{2}\right)}+\frac{4 \pi^{3}}{L^{5}} \times \\
& \times D_{0 I}^{2} \sum_{n=0}^{\infty} n^{3} F_{n I} e_{n I}(t) \int_{L}^{x} \int_{0}^{v} \int_{0}^{w} k_{I, V}\left(y, T_{2}\right) s_{n}(y) d y \frac{d u d v}{D_{I}\left(y, T_{2}\right)}+\int_{L}^{x} \frac{1}{D_{I}\left(v, T_{2}\right)} \int_{0}^{v} k_{I, V}\left(u, T_{2}\right)\left\{\alpha_{2 I}+\int_{L}^{u} \frac{1}{D_{I}(w, T)} \times\right. \\
& \left.\times \frac{4}{L^{2}} \int_{0}^{w} k_{I, V}(y, T) \sum_{n=0}^{\infty} F_{n I} c_{n}(y) e_{n I}(t) \sum_{m=0}^{\infty} F_{m V} c_{m}(y) e_{m V}(t) d y d w-2 D_{0 I} \frac{\pi}{L^{2}} \sum_{n=0}^{\infty} n F_{n I} e_{n I}(t) \int_{L}^{u} \frac{s_{n}(w) d w}{D_{I}\left(w, T_{2}\right)}\right\} \times
\end{aligned}
$$


$\times\left\{\alpha_{2 V}+\frac{4}{L^{2}} \int_{L}^{u} \frac{1}{D_{V}(w, T)} \int_{0}^{w} k_{I, V}(y, T) \sum_{n=0}^{\infty} F_{n I} c_{n}(y) e_{n I}(t) \sum_{m=0}^{\infty} F_{m V} c_{m}(y) e_{m V}(t) d y d w-2 D_{0 I} \frac{\pi}{L^{2}} \sum_{n=0}^{\infty} n F_{n I} \times\right.$

$\left.\times e_{n I}(t) \int_{L}^{u} \frac{S_{n}(w) d w}{D_{I}\left(w, T_{2}\right)}\right\} d u d v$

$V_{2}(x, t)=-D_{0 I} \frac{4 \pi^{2}}{L^{4}} \int_{L}^{x} \int_{0}^{v} \int_{0}^{w} k_{I, V}\left(y, T_{2}\right) \sum_{n=0}^{\infty} n^{2} F_{n I} c_{n}(y) e_{n I}(t) \sum_{m=0}^{\infty} F_{m V} c_{m}(y) e_{m V}(t) d y \frac{w d w}{D_{I}\left(w, T_{2}\right)} \frac{d u d v}{D_{I}\left(v, T_{2}\right)}-$

$-D_{0 V} \frac{4 \pi^{2}}{L^{4}} \int_{L}^{x} \int_{0}^{v} \int_{0}^{w} k_{I, V}\left(y, T_{2}\right) \sum_{n=0}^{\infty} F_{n I} c_{n}(y) e_{n I}(t) \sum_{m=0}^{\infty} m^{2} F_{m V} c_{m}(y) e_{m V}(t) d y \frac{w d w}{D_{I}\left(w, T_{2}\right)} \frac{d u d v}{D_{I}\left(v, T_{2}\right)}+\frac{4 \pi^{3}}{L^{5}} \times$

$\times D_{0 V}^{2} \sum_{n=0}^{\infty} n^{3} F_{n V} e_{n V}(t) \int_{L}^{x} \int_{0}^{v} \int_{0}^{w} k_{I, V}\left(y, T_{2}\right) s_{n}(y) d y \frac{d u d v}{D_{V}\left(y, T_{2}\right)}+\int_{L}^{x} \frac{1}{D_{I}\left(v, T_{2}\right)} \int_{0}^{v} k_{I, V}\left(u, T_{2}\right)\left\{\alpha_{2 I}+\int_{L}^{u} \frac{1}{D_{I}(w, T)} \times\right.$

$\left.\times \frac{4}{L^{2}} \int_{0}^{w} k_{I, V}(y, T) \sum_{n=0}^{\infty} F_{n I} c_{n}(y) e_{n I}(t) \sum_{m=0}^{\infty} F_{m V} c_{m}(y) e_{m V}(t) d y d w-2 D_{0 I} \frac{\pi}{L^{2}} \sum_{n=0}^{\infty} n F_{n l} e_{n I}(t) \int_{L}^{u} \frac{S_{n}(w) d w}{D_{I}\left(w, T_{2}\right)}\right\} \times$

$\times\left\{\alpha_{2 V}+\frac{4}{L^{2}} \int_{L}^{u} \frac{1}{D_{V}(w, T)} \int_{0}^{w} k_{I, V}(y, T) \sum_{n=0}^{\infty} F_{n I} c_{n}(y) e_{n I}(t) \sum_{m=0}^{\infty} F_{m V} c_{m}(y) e_{m V}(t) d y d w-2 D_{0 I} \frac{\pi}{L^{2}} \sum_{n=0}^{\infty} n F_{n I} \times\right.$

$\left.\times e_{n I}(t) \int_{L}^{u} \frac{s_{n}(w) d w}{D_{I}\left(w, T_{2}\right)}\right\} d u d v$

where $\alpha_{2 I}$ and $\alpha_{2 V}$ are determined by the following relations

$\alpha_{2 V}=-\frac{1}{2}\left[\left(L \Theta+S_{I 01}\right)\left(1+\frac{S_{I 10}}{L \Theta}\right)+\frac{S_{I 00}}{L \Theta}\left(W_{V}+S_{V 11}+M_{V 11}\right)+\frac{S_{I 00}}{L \Theta}\left(S_{V 11}+W_{V}+M_{V 111}\right)-\frac{S_{V 00}}{L \Theta} \times\right.$

$\left.\times\left(S_{I 11}+W_{I}+M_{I 11}\right)-\frac{S_{I 10} S_{V 01}}{L \Theta}\right]\left[S_{I 00}\left(1+\frac{S_{V 10}}{L \Theta}\right)-S_{I 10}\left(S_{V 11}+W_{V}+M_{I 11}\right)+\left(1+\frac{S_{I 10}}{L \Theta}\right)\left(S_{I 01}+L \times\right.\right.$

$\left.\times \Theta)-\frac{S_{V 00}}{L \Theta}\left(S_{I 11}+W_{I}+M_{I 11}\right)-S_{V 01} \frac{S_{I 10}}{L \Theta}\right]^{2}\left[\left(1+\frac{S_{V 10}}{L \Theta}\right) S_{I 00}-S_{I 10} S_{V 00}\right]^{-2}-4\left[\left(S_{V 11}+M_{V 11}+\right.\right.$

$\left.\left.\left.+W_{V}\right)\left(1+\frac{S_{I 01}}{L \Theta}\right)-\frac{S_{V 01}}{L \Theta}\left(W_{I}+S_{I 11}+M_{I 11}\right)\right]\left[S_{I 00}\left(1+\frac{S_{V 10}}{L \Theta}\right)-S_{I 10} S_{V 00}\right]^{-1}\right\}^{\frac{1}{2}}$,

$\alpha_{2 I}=-\frac{Q_{I}+S_{I 11}+M_{I 111}+\alpha_{2 V} S_{I 10}}{L \Theta+\alpha_{2 V} S_{I 00}+S_{I 01}}$, 
where

$$
\begin{aligned}
& S_{\rho i j}=\int_{0}^{\Theta} \int_{0}^{L} \frac{x}{D_{\rho}\left(x, T_{2}\right)} \int_{0}^{x} k_{I, V}\left(v, T_{2}\right)\left[\frac{4}{L^{2}} \int_{L}^{v} \int_{0}^{u} k_{I, V}\left(w, T_{2}\right) \sum_{n=0}^{\infty} F_{n I} c_{n}(w) e_{n I}(t) \sum_{m=0}^{\infty} F_{m V} c_{m}(w) e_{m V}(t) \frac{d w d u}{D_{I}\left(u, T_{2}\right)}-\right. \\
& \left.-2 D_{0 I} \frac{\pi}{L^{2}} \sum_{n=0}^{\infty} n F_{n I} e_{n I}(t) \int_{L}^{v} \frac{S_{n}(u) d u}{D_{I}(u, T)}\right]^{i}\left[\frac{4}{L^{2}} \int_{L}^{v} \frac{1}{D_{V}\left(u, T_{2}\right)} \int_{0}^{u} k_{I, V}\left(w, T_{2}\right) \sum_{n=0}^{\infty} F_{n I} c_{n}(w) e_{n I}(t) \sum_{m=0}^{\infty} F_{m V} c_{m}(w) \times\right.
\end{aligned}
$$$$
\left.\times e_{m V}(t) d w d u-2 D_{0 V} \frac{\pi}{L^{2}} \sum_{n=0}^{\infty} n F_{n V} e_{n V}(t) \int_{L}^{v} \frac{S_{n}(u) d u}{D_{V}\left(u, T_{2}\right)}\right]^{j} d w d u d v d x d t
$$$$
W_{I}=4 D_{0 I} \frac{\pi^{2}}{L^{4}} \int_{0}^{\ominus} \int_{0}^{L} \int_{0}^{x} \int_{0}^{u} k_{I, V}\left(w, T_{2}\right) \sum_{n=0}^{\infty} n^{2} F_{n I} c_{n}(w) e_{n I}(t) \sum_{m=0}^{\infty} F_{m V} c_{m}(w) e_{m V}(t) d w \frac{v d u d v}{D_{I}\left(v, T_{2}\right)} \frac{x d x d t}{D_{I}(x, T)}+
$$$$
+4 D_{0 V} \frac{\pi^{2}}{L^{4}} \int_{0}^{\Theta} \int_{0}^{L} \int_{0}^{x} \int_{0}^{u} k_{I, V}\left(w, T_{2}\right) \sum_{n=0}^{\infty} F_{n I} c_{n}(w) e_{n I}(t) \sum_{m=0}^{\infty} m^{2} F_{m V} c_{m}(w) e_{m V}(t) d w \frac{v d u d v}{D_{I}\left(v, T_{2}\right)} \frac{x d x d t}{D_{I}(x, T)}-
$$$$
-2 D_{0 I} \frac{\pi^{3}}{L^{4}} \sum_{n=0}^{\infty} n^{2} F_{n l} e_{n I}(t) \int_{0}^{\theta} \int_{0}^{L} \frac{x}{D_{I}(x, T)} \int_{0}^{x} \frac{v s_{n}(v) d v}{D_{I}\left(v, T_{2}\right)} d x d t
$$$$
W_{V}=4 D_{0 I} \frac{\pi^{2}}{L^{4}} \int_{0}^{\Theta} \iint_{000}^{L x} \int_{I, V}^{u}\left(w, T_{2}\right) \sum_{n=0}^{\infty} n^{2} F_{n I} c_{n}(w) e_{n I}(t) \sum_{m=0}^{\infty} F_{m V} c_{m}(w) e_{m V}(t) d w \frac{v d u d v}{D_{V}\left(v, T_{2}\right)} \frac{x d x d t}{D_{V}(x, T)}+
$$$$
+4 D_{0 V} \frac{\pi^{2}}{L^{4}} \int_{0}^{\Theta} \int_{0}^{L} \int_{0}^{x} \int_{0}^{u} k_{I, V}\left(w, T_{2}\right) \sum_{n=0}^{\infty} F_{n I} c_{n}(w) e_{n I}(t) \sum_{m=0}^{\infty} m^{2} F_{m V} c_{m}(w) e_{m V}(t) d w \frac{v d u d v}{D_{V}\left(v, T_{2}\right)} \frac{x d x d t}{D_{V}(x, T)}-
$$$$
-2 D_{0 V} \frac{\pi^{3}}{L^{4}} \sum_{n=0}^{\infty} n^{2} F_{n V} e_{n V}(t) \int_{0}^{\Theta} \int_{0}^{L} \frac{x}{D_{V}(x, T)} \int_{0}^{x} \frac{v S_{n}(v) d v}{D_{V}\left(v, T_{2}\right)} d x d t
$$ 